\title{
Heralded Entanglement between Atomic Ensembles: Preparation, Decoherence, and Scaling
}

\author{
J. Laurat, K. S. Choi, H. Deng, C. W. Chou, and H. J. Kimble \\ Norman Bridge Laboratory of Physics 12-33, California Institute of Technology, Pasadena, California 91125, USA
}

(Received 26 May 2007; published 2 November 2007)

\begin{abstract}
Heralded entanglement between collective excitations in two atomic ensembles is probabilistically generated, stored, and converted to single-photon fields. By way of the concurrence, quantitative characterizations are reported for the scaling behavior of entanglement with excitation probability and for the temporal dynamics of various correlations resulting in the decay of entanglement. A lower bound of the concurrence for the collective atomic state of $0.9 \pm 0.3$ is inferred. The decay of entanglement as a function of storage time is also observed, and related to the local dynamics.
\end{abstract}

DOI: $10.1103 /$ PhysRevLett.99.180504

Beyond a fundamental significance, quantum control of entanglement between material systems is an essential capability for quantum networks and scalable quantum communication architectures [1,2]. In recent years, significant advances have been achieved in the control of the quantum states of atomic systems, including entanglement of trapped ions [3,4] and between macroscopic spins [5]. By following the seminal paper of Duan, Lukin, Cirac, and Zoller (DLCZ) [6], entanglement between single collective excitations stored in two remote atomic ensembles has also been demonstrated [7]. In the DLCZ protocol, entanglement is created in a probabilistic but heralded way from quantum interference in the measurement process [8-10]. The detection of a photon from one or the other atomic ensemble in an indistinguishable fashion results in an entangled state with one collective spin excitation shared coherently between the ensembles. Such entanglement has been critical for the initial implementation of functional quantum nodes for entanglement distribution [11], for the investigation of entanglement swapping [12] and for lightmatter teleportation [13].

Because of the relevance to quantum networking tasks, it is important to obtain detailed characterizations of the physical processes related to the creation, storage, and utilization of heralded entanglement. Towards this end, significant advances have been demonstrated in the generation of photon-pairs $[14,15]$ and the efficient retrieval of collective excitation [16,17]. Moreover, decoherence processes for a single atomic ensemble in the regime of collective excitation have been investigated theoretically [18] and a direct measurement of decoherence for one stored component of a Bell state recently performed [19]. However, to date no direct study has been reported for the decoherence of an entangled system involving two distinct atomic ensembles, which is a critical aspect for the implementation of elaborate protocols [20-22]. The decoherence of entanglement between ensembles has been shown in recent setups, through the decay of the violation of a Bell inequality [11] and the decay of the fidelity of a teleported state [13]. However, a quantitative analysis was not provided since these setups involved
PACS numbers: 03.67.Mn, 03.65.Yz, 03.67.Hk, 42.50.Dv

many others parameters, such as phase stability over long distances.

In this Letter, we report measurements that provide a detailed and quantitative characterization of entanglement between collective atomic excitations. Specifically, we determine the concurrence $C$ [23] as a function of the normalized degree of correlation $g_{12}$ [16] for the ensembles, including the threshold $g_{12}^{(0)}$ for entanglement $(C>0)$. We also map the decay of the concurrence $C(\tau)$ as a function of storage time for the entangled state, and interpret this decay by measuring the local decoherence on both ensembles taken independently. Compared to Ref. [7], these observations are made possible by a new system that requires no active phase stability and that implements conditional control for the generation, storage, and readout of entangled atomic states.

Our experiment is illustrated in Fig. 1. A single cloud of cesium atoms in a magneto-optical trap is used; two ensembles are defined by different optical paths $1 \mathrm{~mm}$ apart $[11,24]$. This separation is obtained by the use of birefringent crystals close to the cloud, which separate orthogonal polarizations [25]. At $40 \mathrm{~Hz}$, the trap magnetic field is switched off for $7 \mathrm{~ms}$. After waiting $3 \mathrm{~ms}$ for the magnetic field to decay, the two samples are simultaneously illuminated with $30 \mathrm{~ns}$-long and $10 \mathrm{MHz}$ red-detuned write pulses, at a rate of $1.7 \mathrm{MHz}$. Given the duty cycle of the experiment, the effective rate is $180 \mathrm{kHz}$. Spontaneous Raman scattered fields induced by the write beams are collected into single-mode fibers, defining for each ensemble optical modes that we designate as fields $1_{U, D}$ with $50 \mu \mathrm{m}$ waist and a $3^{\circ}$ angle relative to the direction of the write beams $[15,16]$. Fields $1_{U, D}$ are frequency filtered to block spontaneous emission from atomic transitions $|e\rangle \rightarrow|g\rangle$, which do not herald the creation of a collective excitation. After this stage, and before detection, fields $1_{U, D}$ are brought to interfere on a polarizing beamsplitter. A detection event at $D_{1 a, 1 b}$ that arises indistinguishably from either of the fields $1_{U, D}$ projects the atomic ensembles into an entangled state where, in the ideal case, one collective excitation is coherently shared between the $U, D$ ensembles [6,7]. 
(a) Entanglement generation



(b) Entanglement verification

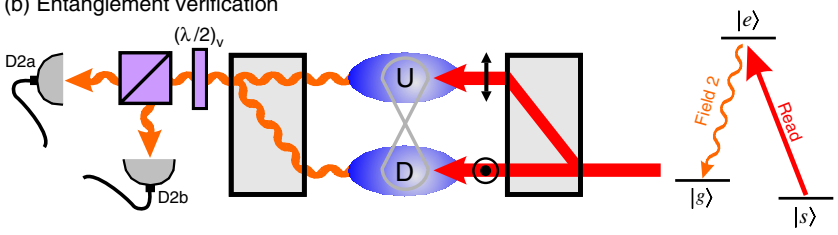

FIG. 1 (color online). (a) A weak write pulse is split into two paths separated by $1 \mathrm{~mm}$ and excites simultaneously two atomic samples, $U, D$. The resulting fields $1_{U, D}$ are combined at the polarizing beam splitter (PBS) and sent to the single-photon detectors $D_{1 a, 1 b}$. A detection event at $D_{1 a}$ or $D_{1 b}$ heralds the creation of entanglement. (b) After a storage time $\tau$, entanglement is verified by mapping the atomic state to propagating fields $2_{U, D}$ by way of read pulses. Tomography is then achieved in two steps, as described in the text. The atomic cloud is initially prepared in the ground state $|g\rangle .\{|g\rangle,|s\rangle,|e\rangle\}$ denote the levels $\left\{\left|6 S_{1 / 2}, F=4\right\rangle,\left|6 S_{1 / 2}, F=3\right\rangle,\left|6 P_{3 / 2}, F=4\right\rangle\right\}$ in atomic Cs. Note that the fields $1_{U, D}$ and $2_{U, D}$ are detected with a small angle relative to the classical beams, which is not represented here for the sake of simplicity.

In the ideal case of small excitation probability, the atom-field 1 joint state can be written for each ensemble:

$$
|\Psi\rangle=\left|0_{a}\right\rangle\left|0_{1}\right\rangle+\sqrt{\chi}\left|1_{a}\right\rangle\left|1_{1}\right\rangle+O(\chi),
$$

with $\left|n_{1}\right\rangle$ the state of the field 1 with $n$ photons and $\left|n_{a}\right\rangle$ the state of the ensemble with $n$ collective excitations. Upon a detection event at $D_{1 a, 1 b}$, in the ideal case, the atomic state is projected into

$$
\left|\Psi_{U, D}\right\rangle=\frac{1}{\sqrt{2}}\left(\left|0_{a}\right\rangle_{U}\left|1_{a}\right\rangle_{D} \pm e^{i \theta}\left|1_{a}\right\rangle_{U}\left|0_{a}\right\rangle_{D}\right)+O(\sqrt{\chi}),
$$

where $\left|0_{a}\right\rangle_{U, D},\left|1_{a}\right\rangle_{U, D}$ refers to the two ensembles $U, D$ with 0,1 collective excitations, respectively [6]. The \pm sign is set by the detector that records the heralding event. The overall phase $\theta$ is the sum of the phase difference of the write beams at the $U$ and $D$ ensembles and the phase difference acquired by fields 1 in propagation from the ensembles to the beam splitter. To achieve entanglement, this phase must be constant from trial to trial [26]. In order to meet this requirement, the initial demonstration reported in [7] employed auxiliary fields to achieve active stabilization for various phases for two ensembles located in distinct vacuum apparatuses. By contrast, in our current setup [Fig. 1(a)], $\theta$ is determined only by the differential phase for the two paths with orthogonal polarizations defined by the birefringent crystals [25]; our small setup has sufficient passive stability without need of adjustment or compensation as the phase does not change by more than a few degrees over $24 \mathrm{~h}$.
To verify operationally entanglement between the $U, D$ ensembles, the respective atomic states are mapped into photonic states by applying simultaneously read pulses in the configuration introduced in Ref. [15], as depicted in Fig. 1(b). The delocalized atomic excitation is retrieved with high efficiency thanks to collective enhancement $[6,16]$ and, in the ideal case, $\left|\Psi_{U, D}\right\rangle$ would be mapped directly to the photonic state of fields $2_{U, D}$ with unity efficiency and no additional components. Stability for the phase difference of the read beams and of fields $2_{U, D}$ is also required in this process; it is again achieved by the passive stability of our current scheme [25]. Since entanglement cannot be increased by local operations [27], the entanglement for the atomic state will always be greater than or equal to that measured for the light fields.

A model-independent determination of entanglement based upon quantum tomography of the fields $2_{U, D}$ has been developed in Ref. [7]. The model consists of reconstructing a density matrix, $\tilde{\rho}_{2_{U}, 2_{D}}$, obtained from the full density matrix by restriction to the subspace with no more than one photon per mode. We also assume that all offdiagonal elements between states with different numbers of photons vanish. The model thus leads to a lower bound for entanglement. As detailed in Ref. [7], $\tilde{\rho}_{2_{U}, 2_{D}}$ can be written in the photon-number basis $|n\rangle|m\rangle$ with $\{n, m\}=$ $\{0,1\}$ as follows:

$$
\tilde{\rho}_{2_{U}, 2_{D}}=\frac{1}{P}\left(\begin{array}{cccc}
p_{00} & 0 & 0 & 0 \\
0 & p_{01} & d & 0 \\
0 & d^{*} & p_{10} & 0 \\
0 & 0 & 0 & p_{11}
\end{array}\right) .
$$

Here, $p_{i j}$ is the probability to find $i$ photons in mode $2_{U}$ and $j$ in mode $2_{D} ; d$ is the coherence term between the $|1\rangle|0\rangle$ and $|0\rangle|1\rangle$ states; and $P=p_{00}+p_{01}+p_{10}+p_{11}$. From $\tilde{\rho}_{2_{U}, 2_{D}}$, one can calculate the concurrence $C$, which is a convenient monotone measurement of entanglement ranging from 0 for a separable state to 1 for a maximally entangled state [23]:

$$
C=\max \left(0, C_{0}\right) \quad \text { with } \quad C_{0}=\frac{1}{P}\left(2|d|-2 \sqrt{p_{00} p_{11}}\right) .
$$

In the regime of low excitation and detection probabilities in which the experiment is performed, the vacuum $p_{00}$ can be approximated by $p_{00} \sim 1-p_{c}$, while the terms $p_{01}$ and $p_{10}$ are given by $p_{10}=p_{01}=p_{c} / 2 . p_{c}$ is the conditional probability of detecting a photon in field 2 from one ensemble following a detection event for field 1 .

Experimentally, we reconstruct $\tilde{\rho}_{2_{U}, 2_{D}}$ and then calculate $C$ by using two configurations for the detection of fields $2_{U, D}$, corresponding to two settings of the $(\lambda / 2)_{v}$ wave plate shown in Fig. 1(b). The diagonal elements of $\tilde{\rho}_{2_{U}, 2_{D}}$ are determined from measurements of the photon statistics for the separated fields $2_{U}, 2_{D}$, i.e., by detecting independently each field. To access the coherence term $d$, fields $2_{U, D}$ are coherently superimposed and the count rates from 
the resulting interference are recorded as a function of the relative phase between the $2_{U, D}$ fields. It can be shown that $d \simeq V\left(p_{10}+p_{01}\right) / 2 \sim V p_{c} / 2$ [7], where $V$ is the visibility of the interference fringe.

To investigate the scaling of entanglement with excitation probability $\chi$, we determine $C$ for various values of $\chi$ for fixed memory time $\tau=200 \mathrm{~ns}$. As $\chi$ increases, higher order terms in the expansion of Eq. (2) cannot be neglected, precisely as in parametric down conversion. A convenient parameter to assess the excitation regime of each ensemble is the normalized intensity cross correlation function $g_{12}$ between field 1 and field 2 [16], defined as $g_{12}=$ $p_{12} /\left(p_{1} p_{2}\right)$ with $p_{12}$ the joint probability for detection events from field 1 and 2 in a given trial and $p_{i}$ the probability for unconditional detections in field $i$. In the ideal case, this function is related to the excitation probability $\chi$ by $g_{12}=1+1 / \chi$, where $g_{12}>2$ defines the nonclassical border in the ideal case [14] and $g_{12} \gg 2$ being the single-excitation regime for the ensembles.

Expressing the two-photon component for the two ensembles as $p_{11}=\chi p_{c}^{2} \sim p_{c}^{2} / g_{12}$, we rewrite the concurrence as

$$
C \simeq \max \left[0, p_{c}\left(V-2 \sqrt{\left(1-p_{c}\right) / g_{12}}\right)\right],
$$

where $g_{12}$ is for either ensemble alone, with $g_{12}^{(U)}=g_{12}^{(D)} \equiv$ $g_{12}$ assumed. The visibility $V$ can be expressed in terms of $g_{12}$ as the higher order terms act as a background noise. With $(1 / 2) p_{1} p_{2}$ a good estimation for the background, the visibility can be written as [25]

$$
V \simeq \xi \frac{p_{12}-p_{1} p_{2}}{p_{12}+p_{1} p_{2}}=\xi \frac{g_{12}-1}{g_{12}+1},
$$

where $\xi$ is the overlap between fields $2_{U, D}$ [28]. In the limit of near zero excitation, as $g_{12}$ goes to infinity, the concurrence reaches its asymptotic value given by the retrieval efficiency $\xi p_{c}$ [29].

Figure 2 presents our measurements of the concurrence $C$ as a function of $g_{12}$. As the excitation probability is decreased, $g_{12}$ increases as does the entanglement. The threshold to achieve $C>0$ is found to be $g_{12}^{(0)} \simeq 7$, corresponding to a probability $p \simeq 1.2 \times 10^{-2}$ per trial for the creation of the heralded entangled state and to a preparation rate $\sim 2 \mathrm{kHz}$. Note that $C=0$ (or $C$ not greater than zero) does not imply that there is no entanglement, only that any possible entanglement is not detected by our protocol, which provides a lower bound for the entanglement. More importantly, in an infinite dimensional Hilbert space, entangled states are dense in the set of all states [30], so that zero entanglement is not provable for an actual experiment by way of the concurrence.

To confirm the model leading to Eq. (6), the inset gives the measured visibility $V$ as a function of $g_{12}$. The solid line is a fit according to Eq. (6) with free parameter $\xi$, leading to an overlap $\xi=0.95 \pm 0.01$, in agreement with the value $\xi=0.98 \pm 0.03$ obtained from an independent two-photon interference measurement. With the fitted

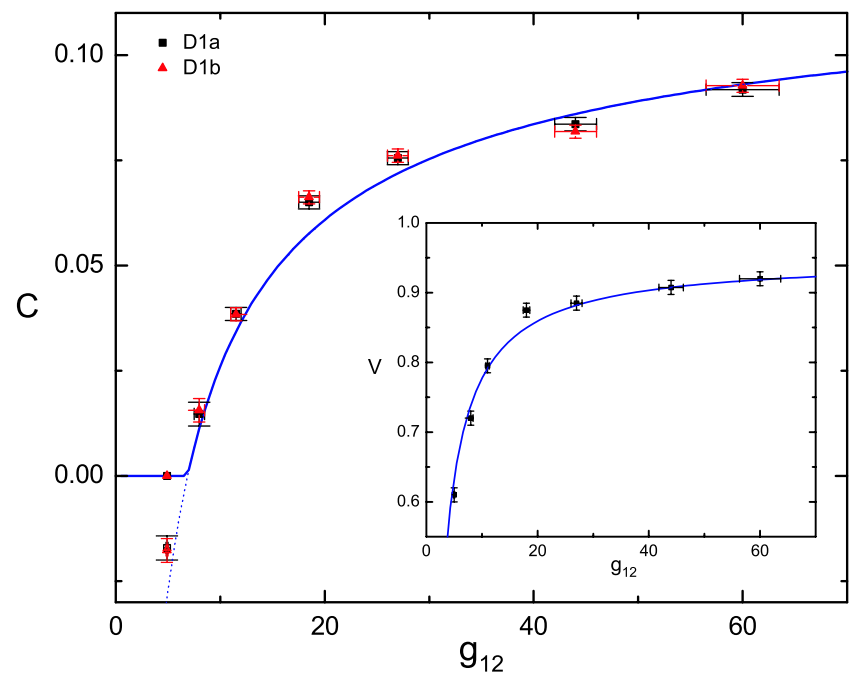

FIG. 2 (color online). Concurrence $C$ as a function of the normalized cross correlation function $g_{12}$, for the two possible heralding events (detection at $D_{1 a}$ or $D_{1 b}$ ). The solid line is obtained from Eq. (5) with the fitted overlap (see inset) and assuming an independently-measured retrieval efficiency at $13.5 \%$. The dotted line corresponds to $C_{0}$. Inset: Average visibility of the interference fringe between the two field- 2 modes. The solid line is a fit using the expression given by Eq. (6), with the overlap $\xi$ fitted to $0.95 \pm 0.01$.

value of $\xi$ and with the independently determined value of the conditional probability $p_{c}=0.135 \pm 0.005$ from measurements performed on each ensemble separately, we compare our measurements of $C$ with the prediction of Eq. (5) (solid line in Fig. 2) and find good agreement.

Table I provides the diagonal elements of the density matrix $\tilde{\rho}_{2_{U}, 2_{D}}$ and the concurrence for the case $g_{12}=60 \pm$ 4 corresponding to a probability to create entanglement $p=9 \times 10^{-4}$ per trial $(160 \mathrm{~Hz})$. A value $C=0.092 \pm$ 0.002 is directly measured at detectors $D_{2 a}, D_{2 b}$ without correction. By way of the independently determined propagation and detection efficiencies, we infer the density matrix $\tilde{\rho}_{2_{U}, 2_{D}}^{\text {output }}$ for fields $2_{U}, 2_{D}$ at the output of the ensembles, from which we obtain a concurrence $C_{2_{U}, 2_{D}}^{\text {output }}=0.35 \pm$ 0.10 . This value exceeds the published state of the art by 2 orders of magnitude [7]. This leap underlines the progress obtained in terms of suppression of the two-

TABLE I. Diagonal elements and concurrence of the density matrices for fields $2_{U, D}$, without and with correction for propagation losses and detection efficiencies. The last column provides the estimated elements and concurrence for the atomic state by considering the readout efficiency $\eta$ at $g_{12}=60 \pm 4$.

\begin{tabular}{lccc}
\hline \hline & $\tilde{\rho}_{2_{U}, 2_{D}}$ & $\tilde{\rho}_{2_{U}, 2_{D}}^{\text {output }}$ & $\tilde{\rho}_{U, D}$ \\
\hline$p_{00}$ & $0.864 \pm 0.001$ & $0.54 \pm 0.08$ & $0 \pm 0.3$ \\
$p_{10}$ & $(6.47 \pm 0.02) \times 10^{-2}$ & $(22 \pm 4) \times 10^{-2}$ & $0.5 \pm 0.15$ \\
$p_{01}$ & $(7.07 \pm 0.02) \times 10^{-2}$ & $(24 \pm 4) \times 10^{-2}$ & $0.5 \pm 0.15$ \\
$p_{11}$ & $(2.8 \pm 0.2) \times 10^{-4}$ & $(3 \pm 2) \times 10^{-3}$ & $0.015 \pm 0.025$ \\
$C$ & $0.092 \pm 0.002$ & $0.35 \pm 0.1$ & $0.9 \pm 0.3$ \\
\hline \hline
\end{tabular}




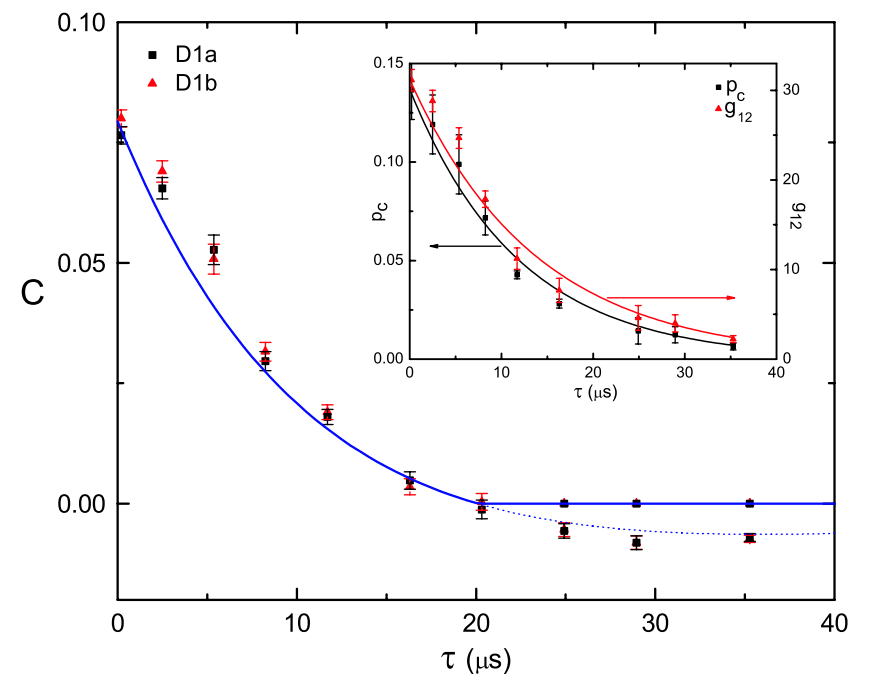

FIG. 3 (color online). Concurrence $C$ as a function of the storage time $\tau$. The solid line is obtained from Eq. (5) assuming the fitted exponential decays, given in the inset, of the individual parameters $p_{c}$ and $g_{12}$ measured independently. The dotted line corresponds to $C_{0}$.

photon component and achievable retrieval efficiency over the past year $[16,17]$. Finally, by way of the conditional readout efficiency $\eta=45 \pm 10 \%$ for mapping of quantum states of the $U, D$ ensembles to the fields $2_{U}, 2_{D}$, we estimate the density matrix $\tilde{\rho}_{U, D}$ and the concurrence $C_{U, D}=0.9 \pm 0.3$ for the collective atomic state. We emphasize that $C_{U, D}$ is an estimate determined from the model developed in Ref. [14] where the fields at the output of the MOT consist of a two-mode squeezed state plus background fields in coherent states.

Turning then to a characterization of the decay of entanglement with storage time $\tau$, we present in Fig. 3 measurements of concurrence $C(\tau)$ for fixed excitation probability $p=1.6 \times 10^{-3}$ corresponding to $g_{12}=30$ at $\tau=200 \mathrm{~ns}$. $C>0$ for $\tau \lesssim 20 \mu \mathrm{s}$, providing a lower bound for the lifetime of entanglement of the ensembles corresponding to $4 \mathrm{~km}$ propagation delay in a fiber.

To investigate the dynamics in Fig. 3, the inset shows the decay of the average $g_{12}$ and conditional probability $p_{c}$ for the ensembles taken independently. Such local decoherence has been investigated as the result of inhomogeneous broadening of the Zeeman ground states due to residual magnetic fields [18,19,31]. Our current measurement shows the effect of this local decoherence on the entanglement of the joint system of the ensembles. For this purpose, our measurements of $C$ are superposed with a line $C(\tau)$ given by Eq. (5), where the fitted exponential decay for $p_{c}(\tau), g_{12}(\tau)$ (with similar decay $\left.\simeq 13 \mu \mathrm{s}\right)$ and the overlap $\xi$ determined in Fig. 2 are employed. The agreement evidenced in Fig. 3 confirms the principal role of local dephasing in the entanglement decay.

In conclusion, we have reported a detailed study of the behavior of entanglement between collective excitations stored in atomic ensembles, including the dependence of the concurrence on the degree of excitation and the quantitative relationship of local decoherence to entanglement decay. The temporal dynamics reveal a finite-time decay, with separability onset for storage time $\tau \sim 20 \mu \mathrm{s}$. From a more general perspective, the inferred concurrence for the collective atomic state, $C_{U, D}=0.9 \pm 0.3$, is comparable to values obtained for entanglement in the continuous variable regime [32] and for entanglement of the discrete internal states of trapped ions $[3,4]$.

We acknowledge our collaboration with S. J. van Enk and discussions with D. Felinto and H. de Riedmatten. This research is supported by the DTO and by the NSF. J.L. acknowledges financial support from the EU (Marie Curie fellowship) and H. D. from the CPI at Caltech.

[1] P. Zoller et al., Eur. Phys. J. D 36, 203 (2005).

[2] H. J. Briegel et al., Phys. Rev. Lett. 81, 5932 (1998).

[3] H. Häffner et al., Appl. Phys. B 81, 151 (2005).

[4] C. Langer et al., Phys. Rev. Lett. 95, 060502 (2005).

[5] B. Julsgaard, A. Kozhekin, E. S. Polzik, Nature (London) 413, 400 (2001).

[6] L. M. Duan et al., Nature (London) 414, 413 (2001).

[7] C. W. Chou et al., Nature (London) 438, 828 (2005).

[8] R. H. Dicke, Am. J. Phys. 49, 925 (1981).

[9] C. Cabrillo et al., Phys. Rev. A 59, 1025 (1999).

[10] S. Bose et al., Phys. Rev. Lett. 83, 5158 (1999).

[11] C. W. Chou et al., Science 316, 1316 (2007).

[12] J. Laurat et al., New J. Phys. 9, 207 (2007).

[13] Y.-A. Chen et al., arXiv:0705:1256.

[14] C. W. Chou et al., Phys. Rev. Lett. 92, 213601 (2004).

[15] V. Balić et al., Phys. Rev. Lett. 94, 183601 (2005).

[16] J. Laurat et al., Opt. Express 14, 6912 (2006).

[17] J. K. Thompson et al., Science 313, 74 (2006).

[18] D. Felinto et al., Phys. Rev. A 72, 053809 (2005).

[19] H. de Riedmatten et al., Phys. Rev. Lett. 97, 113603 (2006).

[20] L. Jiang, J. M. Taylor, and M. D. Lukin, Phys. Rev. A 76, 012301 (2007).

[21] Z.-B. Chen et al., Phys. Rev. A 76, 022329 (2007).

[22] B. Zhao et al., Phys. Rev. Lett. 98, 240502 (2007).

[23] W. K. Wootters, Phys. Rev. Lett. 80, 2245 (1998).

[24] D. N. Matsukevich and A. Kuzmich, Science 306, 663 (2004).

[25] C. W. Chou, Ph.D. thesis, California Institute of Technology.

[26] S. J. van Enk, N. Lutkenhaus, and H. J. Kimble, Phys. Rev. A 75, 052318 (2007).

[27] C. H. Bennett et al., Phys. Rev. A 54, 3824 (1996).

[28] D. Felinto et al., Nature Phys. 2, 844 (2006).

[29] An alternative approach is to determine the suppression of two-photon events relative to the square of the probability of single-photon events for the fields $2, h \equiv p_{11} /\left(p_{10} p_{01}\right)$. $h<1$ is a necessary condition for entanglement $[7,11,12]$. Here, for $g_{12}=60, h=0.060 \pm 0.005$.

[30] J. Eisert, C. Simon, and M. Plenio, J. Phys. A 35, 3911 (2002).

[31] D. Matsukevich et al., Phys. Rev. Lett. 96, 033601 (2006).

[32] J. Laurat et al., Phys. Rev. A 71, 022313 (2005). 